studies assessing its sensitivity to change will inform whether MVF can be used as end point of skin microvascular involvement in SSc.

References:

[1] C. Tolosa-Vilella et al. Semin Arthritis Rheum 46(2016):200-208.

Disclosure of Interest: None declared

DOI: 10.1136/annrheumdis-2017-eular.6582

\section{THU0660 POLYMYALGIA RHEUMATICA TREATMENT SERUM BIOMARKERS VERSUS RHEUMATOID ARTHRITIS}

M.K. Meyer ${ }^{1}$, M. Andersen ${ }^{1}$, T.V. Stausbo ${ }^{2}$, K.J. Elbæk ${ }^{2}$, G.N. Andersen ${ }^{1}$ A. Stensballe ${ }^{2}$. ${ }^{1}$ Department of Rheumatology, Nortmark Regional Hospital, Hjorring; ${ }^{2}$ Department of Health Science and Technology, Aalborg University, Aalborg, Denmark

Background: Polymyalgia rheumatica (PMR) is a systemic inflammatory disorder with unknown etiology and overlapping symptoms with giant cell arthritis and late-onset rheumatoid arthritis (RA). To date, no proteomics studies have been performed on PMR patients, and the number of biomarker studies remain limited. Objectives: The primary aim of this study was to thoroughly investigate the corticosteroid treatment serum proteome of PMR with a focus on acute-phase reactions, complement system, and cytokines.

Methods: Filter-aided sample preparation for mass spectrometry, cell free DNA (cfDNA) assay, and 10plex cytokine assay were applied to PMR serum samples from the same patients before and after treatment, DMARD-naïve RA patients, and healthy controls.

Results: The core serum proteomes of the four groups were remarkably similar, and consisted of $>200$ proteins, which included acute-phase reactants, coagulation and complement proteins (figure), immunoglobulins, and apolipoproteins, and several more. Acute Phase Serum Amyloid A (SAA1) was differentially less abundant after PMR treatment, and CRP (after adjusting for two patients with low baseline CRP). cfDNA were more abundant in both groups of PMR compared to healthy controls. Complement factors were narrowly distributed and not affected by PMR treatment. The individual serum proteome of each PMR patient provided more than 100 differentially abundant proteins, and highlights the heterogeneity of patients.

Polymyalgia Complement Factors Before and After treatment
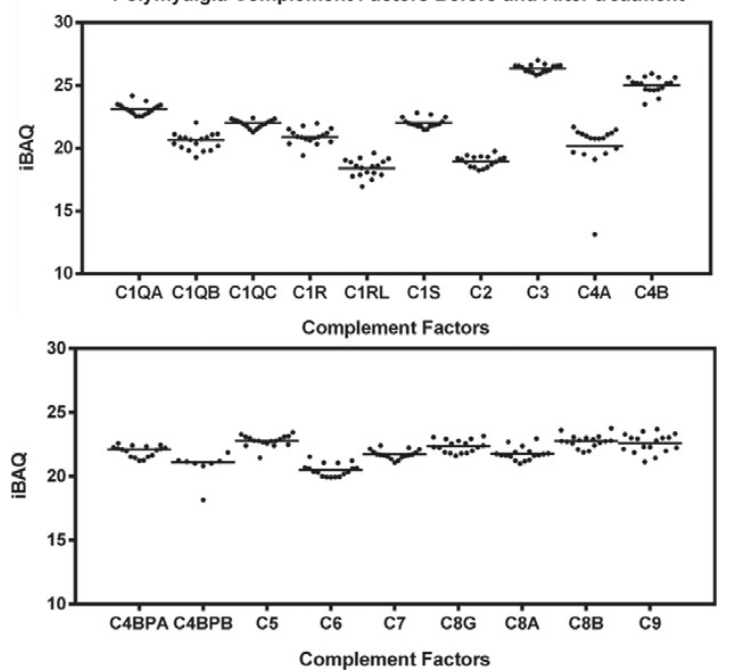

Conclusions: We have established the core serum proteome of PMR in response to treatment, and compared it with RA and healthy controls. The results suggest a functional role of SSA1, and increased cfDNA in the pathogenesis of PMR indicates the activation of NETs.

Disclosure of Interest: None declared

DOI: 10.1136/annrheumdis-2017-eular.5844

\section{THU0661 CLINICAL RELEVANCE OF DETECTING ANTI-ADALIMUMAB ANTIBODIES WITH A DRUG-TOLERANT ASSAY}

A. Martinez-Feito $^{1}$, L.Y. Bravo Gallego ${ }^{2}$, B. Hernandez-Breijo ${ }^{1}$, C. Plasencia ${ }^{1}$ A. Jochems ${ }^{1}$, M.A. Gonzalez ${ }^{2}$, I. Monjo ${ }^{1}$, D. Peiteado ${ }^{1}$, G. Bonilla ${ }^{1}$, P. Nozal ${ }^{2}$,

A. Balsa ${ }^{1}$, D. Pascual-Salcedo ${ }^{1}$. ${ }^{1}$ Immuno-Rheumatology research group;

2 Immunology, University Hospital la Paz, madrid, Spain

Background: Adalimumab (Ada) has proven effective in treating rheumatoid arthritis (RA) and spondyloarthropathies ( $\mathrm{SpA}$ ), although approximately $30 \%$ of responders will present secondary clinical failure. Immunocomplex formation between antibodies to Ada (ATA) and Ada can increase drug clearance. Most of the assays to measure ATA present drug interference. Currently, different assays are available to measure total ATA (free and complexed).

Objectives: To compare the detection of ATA along Ada treatment between two assays: one drug-sensitive and another drug-tolerant assay. Study the association of ATA with the clinical status.

Methods: This is a prospective observational study with 63 patients with rheumatic diseases under Ada treatment enrolled at Biological Therapy Unit of Hospital La Paz. Serum Ada levels were measured by ELISA and serum ATA levels by two assays: an in-house two-site (bridging) ELISA (bELISA) to detect uncomplexed ATA (free ATA) and a drug-tolerant assay developed by ImmundiagnostikÒ (Bensheim, Germany) (IDK) to measure simultaneously free and complexed ATA (total ATA). Samples were evaluated at 0, 0.2, 0.5, 1, 1.5 and 2 years after Ada initiation.

Results: Out of the 63 studied patients, $12(19 \%)$ had RA and the rest $(51$, $81 \%$ ) had different spondyloarthropathies (24, 38\%) ankylosing spondylitis, 9 $(14 \%)$ psoriatic arthritis, $14(22 \%)$ undifferentiated spondyloarthritis and $4(6 \%)$ spondyloarthritis associated with inflammatory bowel disease). Thirty-one patients (49\%) received concomitant methotrexate (26\% RA patients and $74 \% \mathrm{SpA}$ patients), $13(21 \%)$ received another DMARDs associated to Ada and 19 (30\%) were on monotherapy.Out of the 63 patients, in 27 (43\%) no ATA were detected. Thirty six patients $(57 \%)$, were IDK+ and 12 patients $(19 \%)$ were bELISA+ (all of them IDK+). The presence of ATA by bELISA was associated with absence of serum Ada levels. However, most (78\%) samples with complexed ATA had low circulating Ada levels $(1.65 \mathrm{mg} / \mathrm{ml}$ in ATA+ vs $6.25 \mathrm{mg} / \mathrm{ml}$ in ATA-, $\mathrm{p}<0.0001)$. ATA appeared by IDK at earlier treatment stages than by bELISA, statistically different at all studied time points.In patients who dropped-out (30 patients, $48 \%$ ) ATA detection was frequent and significant by both methods. Ten patients $(33 \%)$ bELISA ATA+ dropped-out vs 2 patients bELISA ATA+ in those who continued treatment $(p<0.0001)$. Twenty four patients $(80 \%)$ IDK ATA+ dropped-out vs 12 patients $(36 \%)$ who continued treatment $(p<0.0001)$. The percentage of ATA detected by IDK was higher than by bELISA, with a tendency of more IDK ATA+ among patients with less survival $(24,80 \%$ IDK positive vs $10,33 \%$ bELISA +; $\mathrm{p}=0.06$ )

Conclusions: ATA are detected by a drug-tolerant assay at earlier stages of treatment than by bELISA. The antibodies formed early are associated with lower levels of circulating Ada, indicating higher drug clearance. This information might be useful to implement the Therapeutic Drug Monitoring. However, the detection of early complexed ATA has not demonstrated a significant advantage over the bELISA to predict treatment survival.

Acknowledgements: This work has been supported by a collaboration with Leti laboratories.

Disclosure of Interest: None declared

DOI: 10.1136/annrheumdis-2017-eular.2864

\section{THU0662 COMPARING ELECTRONIC COLLECTION OF PATIENT REPORTED OUTCOMES AT HOME VERSUS TOUCH-SCREENS IN THE WAITING AREA AMONG PATIENTS WITH ARTHRITIS IN CLINICAL PRACTICE: A RANDOMISED AGREEMENT STUDY WITH ONLINE RECRUITMENT USING DANBIO}

A.E. Secher $^{1}$, B. Glintborg ${ }^{1}$, H. Gudbergsen ${ }^{2}$, N.S. Krogh ${ }^{3}$, D.V. Jensen ${ }^{1}$, I.J. Sørensen ${ }^{1}$, R. Christensen ${ }^{2}$, M. Skougaard ${ }^{2}$, M.L. Hetland ${ }^{1}$. ${ }^{1}$ DANBIO registry, Glostrup; ${ }^{2}$ The Parker Institute; ${ }^{3}$ Zitelab Aps, Frederiksberg, Denmark

Background: Collection of patient-reported outcomes (PROs) is an important aspect of modern treatment strategies. Electronic capture in waiting areas by touch screens is part of routine care in the Danish DANBIO registry ${ }^{1}$. It is not known whether this data collection can be replaced with electronic data entry from home.

Objectives: To test the feasibility of online patient recruitment via touch screens and to investigate if electronic reporting of PROs from home (ELECTOR ITplatform) is comparable to reporting completed at the hospital among patients with rheumatoid arthritis (RA) or axial spondyloarthritis (AS).

Methods: Patients with RA or AS were recruited via touch screens and randomized to one of two groups by a pre-computed list generated through DANBIO; the first group completing the PROs at home and then at the hospitalsite, and vice versa for the second group. All patients completed the Health Assessment Questionnaires (HAQ), the Visual Analogue Scales (VAS) for fatigue, pain and global health and the annual visit questions. Furthermore, AS patients completed the Bath Ankylosing Spondylitis Disease Activity Index (BASDAI) and the BAS Function Index (BASFI). Pearson's Chi-square test, independent samples t-test and Mann-Whitney U test were calculated. Smallest detectable differences (SDDs), 95\% confidence intervals (Cls) and intra-class correlation coefficients (ICCs) were calculated.

Clinicaltrials.gov identifier: NCT02818478.

Results: A total of 952 patients received invitation, 45\% accepted, 127 patients were contacted by phone, and $56(44 \%)$ gave consent to participate. 22 patients with RA and 20 patients with AS completed the trial.

All differences between scores from home vs hospital were smaller than SDD, and all were non-significant $(\mathrm{p}<0.05)$, except for BASFI and BASDAI item 5 ICC ranged from $0.947-0.990(p<0.00001)$. Annual visit questions showed $96 \%$ concordance between the two methods. $50 \%$ of the patients preferred from home data entry over hospital and $10 \%$ vice versa.

Conclusions: Recruitment of patients for a randomized trial via touch screens was feasible. PROs collected from patients' own devices at home generated 